\title{
SOME RECENT CONTRIBUTIONS TO ALGEBRAIC GEOMETRY*
}

\section{BY VIRGIL SNYDER}

1. Introduction. During the last decade mathematical literature has been enriched by over a thousand contributions to algebraic geometry, including about eight hundred to the narrower field of rational transformations. It would be an ambitious task to report on this immense field in one short talk. Today I wish to speak of only three problems, each one somewhat well defined, or even narrow. Of these, one furnishes a striking example of mathematical elegance in providing one solution to what was regarded as several distinct problems. The other two are capable of unlimited extension, each new enlarged field furnishing phases not existing in the earlier ones.

2. Series of Composition of Veneroni Transformations. The Cremona transformations determined by a system of bilinear equations between the systems of coordinates of the two associated spaces were among the first to be considered in $S_{2}$ and $S_{3}$. For the general case the configuration of fundamental and principal elements can be at once expressed in terms of the vanishing of certain determinants of a matrix. (Segre. $\dagger$ ) This has recently been generalized to spaces of higher dimensions by various authors, in particular to $S_{n}$ by Godeaux. $\ddagger$ Numerous particular cases have been considered. If $x_{i} x_{i}^{\prime}=x_{k} x_{k}^{\prime}$, the extreme case of inversion results. Transformations made up of this inversion and of collineations have no fundamental curves of the first kind; they have a series of composition somewhat similar to that of the general case for $S_{2}$.

If in every bilinear equation the coefficients $a_{i k}$ and $a_{k i}$ are equal, the transformation is involutorial and can be expressed in terms of polarity as to a series of quadrics or as to null systems. In case all the polarities are quadric and the quadric primals are independent, there are only a finite number of invariant

* Presented to the Society at the symposium held in New York, March 30, 1934 , at the invitation of the program committee.

† C. Segre, Rendiconti dei Lincei, (2), vol. 9 (1900), pp. 253-260.

$\ddagger$ L. Godeaux, Lombardo Istituto Rendiconti, (2), vol. 43 (1910), pp. 116-119. 
points. These have been discussed by M. del Re.* The case of $n=4$ has been investigated by Miss Alderton, $\dagger$ and various further properties have been given by Wong $\ddagger$ and Roth. $\S$

The general Cremona transformation for $S_{r}, r>2$, does not have a series of composition, that is, it cannot be expressed as the product of transformations of lower order.

A Cremona transformation in $S_{3}$ of order $n=4 k+r$ having as base curve of maximal multiplicity a sextic curve $C$ of genus $p=3$ and of multiplicity $k+1$, is the product of a $(3,3)$ and of another transformation of order $n$. (Piazzolla-Beloch. $\|$ )

Similarly, a $T_{2 n-1}: C_{3}{ }^{(n-1)}$, the only other base-system consisting of bisecants of $C_{3}$, has a definite series of composition. (Montesano. T) This case has been developed further by Tinto.**

3. Case $n=3$. The most important particular case from this point of view for $n=3$ is that in which the base $C_{6}$ is composed of four skew lines $r_{i}$ and their two transversals $u, v$. The inverse is defined by cubic surfaces through four skew lines $r_{i}^{\prime}$ and their two transversals $u^{\prime}, v^{\prime}$. A point on $r_{i}$ has for image a line meeting three lines $r_{k}^{\prime}$, so that $r_{i}$ itself is transformed into the quadric through three lines $r_{k}^{\prime}$. A point on $u$ (or $v$ ) has the whole line $u^{\prime}$ (or $v^{\prime}$ ) for image, and conversely. These transversals are simple fundamental lines of the second kind.

The image of an arbitrary line is a space cubic meeting each $r_{i}^{\prime}$ twice, but not meeting $u^{\prime}$ or $v^{\prime}$. A line $r$ meeting $u$ and $v$ is transformed into a line meeting $u^{\prime}$ and $v^{\prime}$. The $(1,1)$ correspondence between this line and its image is a collineation, such

* Maria del Re, Naples Rendiconti, (3), vol. 28 (1922), pp. 203-211, and (3), vol. 35 (1929), pp. 208-217.

† Nina Alderton, University of California Publications in Mathematics, vol. 1 (1923), pp. 345-358.

$\ddagger$ B. C. Wong, Annals of Mathematics, (2), vol. 27 (1926), pp. 330-336, and vol. 28 (1927), pp. 251-262; American Journal of Mathematics, vol. 49 (1927), pp. 383-388; this Bulletin, vol. 35 (1929), pp. 829-832.

$\S \mathrm{L}$. Roth, Proceedings of the Cambridge Philosophical Society, vol. 29 (1933), pp. 178-183.

\| M. Piazzolla-Beloch, Annali di Matematica, (3), vol. 16 (1909), pp. 27-98.

I D. Montesano, Naples Rendiconti, (3), vol. 27 (1921), pp. 116-127 and 164-175.

** F. J. Tinto, Proceedings of the Edinburgh Mathematical Society, vol. 34 (1916), pp. 133-145. 
that the points $r, u$ and $r, v$ are transformed in to $r^{\prime}, u^{\prime}$ and $r^{\prime}, v^{\prime}$. Through a point $P$ in $(x)$ passes a single line meeting $u, v$; through its image $P^{\prime}$ passes a single line meeting $u^{\prime}, v^{\prime}$. The collineation is now uniquely fixed. Let $u$ be taken as $x_{1}=0, x_{2}=0$ and $v$ as $x_{3}=0, x_{4}=0$. For $r_{1}, r_{2}$ the lines $x_{1}=0, x_{3}=0$ and $x_{2}=0$, $x_{4}=0$ may be taken. Then for $r_{3}$ take $a x_{1}+b x_{2}=0, c x_{3}+d x_{4}=0$ and $r_{4}^{\prime}$ is $a^{\prime} x_{1}+b^{\prime} x_{2}=0, c^{\prime} x_{3}+d^{\prime} x_{4}=0$.

Since the quadric surface through any three lines $r_{i}$ contains the two transversals, a quadric and any plane of the pencil through the residual line will be a cubic surface of the homaloidal system. If $H_{13}=0$ is the equation of the quadric through all the lines except $x_{1}=0, x_{3}=0$, etc., we may write

$$
x_{1}=x_{1} H_{13}, \quad x_{2}^{\prime}=x_{2} H_{24}, \quad x_{3}^{\prime}=x_{3} H_{13}, \quad x_{4}^{\prime}=x_{4} H_{24} .
$$

Similar pencils are of the forms

$$
\begin{gathered}
\left(a x_{1}+b x_{2}\right) H_{a, c}+\left(c x_{3}+d x_{4}\right) H_{a, c}=0, \\
\left(a^{\prime} x_{1}+b^{\prime} x_{2}\right) H_{a^{\prime}, c^{\prime}}+\text { etc. }=0 .
\end{gathered}
$$

By writing the equations of $H_{13}, H_{24}$ we can now obtain the forms of the defining bilinear equations

$$
\begin{gathered}
x_{1}^{\prime} x_{3}-x_{1} x_{3}^{\prime}=0, \quad x_{2}^{\prime} x_{4}-x_{2} x_{4}^{\prime}=0, \\
\left(c d^{\prime}\right)\left(a x_{1}+b x_{2}\right)\left(b^{\prime} x_{3}^{\prime}-a^{\prime} x_{4}^{\prime}\right) \\
-\left(a b^{\prime}\right)\left(d^{\prime} x_{1}^{\prime}-c^{\prime} x_{2}^{\prime}\right)\left(c x_{3}+d x_{4}\right)=0 .
\end{gathered}
$$

4. Products of $(3,3)$ Cremona Transformations. Now consider the product of $T_{1}: r_{i} u, v ; r_{i}^{\prime} u^{\prime}, v^{\prime}$ and $T_{2}: r_{i}^{\prime} u^{\prime}, v^{\prime} ; r_{i}^{\prime}, u^{\prime \prime}, v^{\prime \prime}$ in which the same lines $u^{\prime}, v^{\prime}$ appear in $T_{1}^{-1}$ and in $T_{2}$. The lines $r_{i}^{\prime}, r_{i}^{\prime}$ may all be distinct or in part the same. Similarly for the continued product $T_{1}, T_{2}, \cdots, T_{k}$. For every value of $k$ the transformation is determined by two lines $u, v$ of the same multiplicity and of a number of lines, of various multiplicities, meeting both of them, and of no other base elements.

Conversely, consider a transformation $T$ defined by the two properties:

(a) To a line $r$ meeting two skew lines $u$, $v$ shall correspond a line $r^{\prime}$ meeting two skew lines $u^{\prime}, v^{\prime}$.

(b) The projectivity established between $r$ and $r^{\prime}$ shall never be degenerate, and the point $r, u$ shall correspond to the point $r^{\prime}, u^{\prime}$; similarly for $r, v$ and $r^{\prime}, v^{\prime}$. 
The transformations determined by these conditions have $u, v$ to the same multiplicity $\nu$; a number of lines meeting both are base elements of the web, and there can be no other base elements. The transformation is of order $2 \nu+1$. We may then write

$$
\begin{aligned}
& \left|S_{2}\right| \sim\left|\phi_{2 \nu} \phi_{2 v+1}\right|: u^{\nu} v^{\nu} r_{1} \ldots r_{p}^{\rho_{p}}, \\
& \left|S_{1}\right| \sim\left|C_{2 \nu} C_{2 \nu+1}\right|: r_{1}^{2 \rho_{1}} \ldots r_{p}^{2 \rho_{p}} .
\end{aligned}
$$

A plane through $u$ (or $v$ ) is transformed into a ruled surface $R_{2 \nu+1}: u^{\prime \nu} v^{\prime \nu+1} \gamma^{2 \rho_{1}} \ldots r_{p}^{2 \rho_{p}}$.

Each line $r_{i}$ is transformed into a ruled surface; each point of $r_{i} \sim$ a rational curve $o^{\prime}$ of order $\rho_{i}{ }^{\prime}$, not meeting $u^{\prime}, v^{\prime}$, which generates $R_{2} \rho_{i}: u^{\prime} \rho_{i}{ }^{\prime} v^{\prime} \rho_{i}{ }^{\prime}$.

An arbitrary plane $\omega$ meets $u$, $v$ each in a point and contains the line $l$ joining them. Let $\omega^{\prime}$ be any plane (not containing $\left.u^{\prime}, v^{\prime}\right)$ through the image of this line. Given any point $T$ in $\omega$, through it passes a unique line $c$ meeting $u, v$. The image line $c^{\prime}$ meets $u^{\prime}, v^{\prime}$ and pierces $\omega^{\prime}$ in $T^{\prime}$. Between $\omega, \omega^{\prime}$ exists a plane Cremona transformation. Let $T$ describe a line $p$ in $\omega$. The lines $u, v, p$ determine a quadric surface, the image of which is a surface of order $4 \nu+2$. The section by $\omega^{\prime}$ contains $l^{\prime}$ and the image of $p$, hence

$$
c_{1} \sim c_{4 \nu+1}^{\prime}:{U^{\prime 2 \nu+1}}^{{ }^{\prime 2 \nu+1}} \sum_{i=1}^{p^{\prime}} R_{i}^{\rho_{i^{\prime}}} .
$$

Since in a plane Cremona transformation the number of $F$ points in the two planes is the same, it follows that $p=p^{\prime}$.

From the two fundamental relations of a plane Cremona transformation, we have further $\sum \rho_{i}=4, \sum \rho_{i}{ }^{2}=2 \nu(\nu+1)$. Let $\rho_{1} \geqq \rho_{2} \geqq \cdots \geqq \rho_{p}$. Then

$$
\begin{aligned}
\rho_{1}^{2}+\rho_{2}^{2}+\rho_{3}^{2}+\rho_{4}\left(\rho_{4}+\cdots+\rho_{p}\right) & \geqq 2 \nu(\nu+1), \\
\rho_{1}^{2}+\rho_{2}^{2}+\rho_{3}^{2}+\rho_{4}\left(4 \nu-\rho_{1}-\rho_{2}-\rho_{3}\right) & \geqq 2 \nu(\nu+1), \\
\nu\left(\rho_{1}-\rho_{4}\right)+\nu\left(\rho_{2}-\rho_{4}\right)+\nu\left(\rho_{3}-\rho_{4}\right)+4 \nu \rho_{4} & \geqq 2 \nu(\nu+1), \\
\rho_{1}+\rho_{2}+\rho_{3}+\rho_{4} & \geqq 2(\nu+1) .
\end{aligned}
$$

From this last equation it follows that if the web of surfaces, homaloids of the planes of space, is transformed by means of the $(3,3)$ transformation defined by $r_{1}, r_{2}, r_{3}, r_{4}$, the order of the surfaces of the web will be lowered. By continuing this process it follows that every transformation of this kind can be expressed as the product of $(3,3)$ transformations defined by four skew 
base lines. (Montesano.*) The complete table of characteristics can now be constructed, and all the details of incidence determined exactly as for a plane Cremona transformation.

5. Case $n=4$. In $S_{4}$, consider five planes $\pi_{i}$, no two lying in an $S_{3}$. The system $\left|\phi_{3}{ }^{4}\right|$ of quartic primals passing through them form a system $\infty^{4}$ of homaloids. The base $M_{2}{ }^{10}$ consists of the five planes $\pi_{i}$ and of a ruled surface $R_{5}$ formed by the $\infty^{1}$ lines which meet all five planes. Lines meeting any four of the planes $\pi_{i}$ are all $P$ lines. They generate a Segre 10 nodal cubic primal $V_{3}{ }^{3}, \psi_{i}: 4 \pi_{k}$, not $\pi_{i}$. This cubic primal and any $S_{3}$ through $\pi_{i}$ together form a quartic primal of the homaloidal system. The equations of the associated Cremona transformation therefore become $x_{i}^{\prime}=\psi_{i} S_{3, i}: \pi_{i}$. Each $\psi_{i}$ contains $\infty^{2}$ lines, images of the points of $\pi_{i}$. The inverse system is of the same form as the given one. Any two of the base planes, as $\pi_{1}, \pi_{2}$, meet in a point $\pi_{12}$. The plane through $\pi_{12}, \pi_{23}, \pi_{31}$ meets $\pi_{1}, \pi_{2}, \pi_{3}$ each in a line lying on $\psi_{4}$ and $\psi_{3}$. This plane is transformed into the point $P_{45}^{\prime}$ common to $\pi_{4}^{\prime}, \pi_{5}^{\prime}$; it is a double point on $\psi_{1}^{\prime}, \psi_{2}^{\prime}, \psi_{3}^{\prime}$.

The primals $\psi_{4}, \psi_{5}$ intersect in $\pi_{1}, \pi_{2}, \pi_{3}$ and the plane $\pi_{45}$ determined by $\pi_{12}, \pi_{23}, \pi_{31}$, and $R_{5}$. A section by any $S_{3}$ consists of three skew lines, a line meeting all of them, and of a quintic curve which is therefore elliptic. The base $R_{5}$ is an elliptic ruled surface. Every generator of $R_{5}$ is a base line and a $P$ line having a line in $\left(x^{\prime}\right)$ for image in such a way that any point on either line has the whole other line for image, $R_{5} \sim R_{5}^{\prime}$.

Two primals $\phi$ of the homaloidal system intersect in a surface of order 16, consisting of $5 \pi_{i}, R_{5}$, and a variable surface $f$ of order 6. A section of $f$ by an $S_{3}$ is a sextic curve $C_{1}$ of $p=3$, meeting $R_{5}$ in 5 points. The surface $f$ meets each $\pi_{i}$ in an elliptic cubic curve, and $R_{5}$ in 5 generators. Three primals $\phi$ not belonging to the same pencil meet in a normal $C_{4}$ which has three points in each base $\pi_{i}$. Since a line in $(x)$ is uniquely fixed by two points on it, it follows that a unique $C_{4}$ can be passed through two points and have five given planes of $S_{4}$ for trisecants. (Veneroni, $\uparrow$ White, $\ddagger$ Todd. $\S$ ) Consider the $\infty^{6}$ lines of

* D. Montesano, Naples Atti, (2), vol. 18, (1930), 44 pp.

$\dagger$ E. Veneroni, Lombardo Istituto Rendiconti, vol. 34 (1901), pp. 640-644.

$\ddagger$ F. P. White, Proceedings of the Cambridge Philosophical Society, vol. 21 (1922), pp. 216-227.

§ A. J. Todd, ibid., vol. 26 (1930), pp. 323-333. 
$S_{4} ; \infty^{6}\left|C_{4}\right|$ have 5 given planes for trisecants. Of these, $\infty^{3}$ pass through a given point, hence a finite number have a sixth plane for trisecant, images of lines meeting $f$ (image of the sixth plane) in three points. This number is one (White*); hence a normal $C_{4}$ can be passed through a given point, having six given planes for trisecants. It was seen that $R_{5}$ is cut by $S_{5}$ in a $C_{5}$, $p=1$; hence an $S_{3}$ through a generator $g$ of $R_{5}$ will contain an elliptic $C_{4}$, and an $S_{3}$ through two generators meets $R_{5}$ in an elliptic cubic, hence a plane curve. There are $\infty^{2}$ pairs of generators on $R_{5}$, but if $\pi$ is a plane containing a cubic curve $\gamma_{3}$ on $R_{5}$, through $\pi$ can be passed $\infty^{1}\left|S_{3}\right|$, each containing a pair of generators of $R_{5}$; hence $R_{5}$ contains $\infty^{1}$ plane cubic curves $\gamma_{3}$. The base planes $\pi_{i}$ are among the planes $\pi$ containing curves $\gamma_{3}$. Any plane $\pi$ meeting $R_{5}$ in $\gamma_{3}$ is transformed into a plane $\pi^{\prime}$, since through every point of $\gamma_{3}$ passes a generator $g$ of $R_{5}$; hence $R_{5}{ }^{\prime}$ is a component of the image $f^{\prime}$ of this plane.

Let $\pi, \pi^{\prime}$ be a pair of associated planes in $T_{4,4}$. Each contains a cubic curve $\gamma_{3}, \gamma_{3}^{\prime}$ and these curves are projectively equivalent. A line $l$ in $\pi$ meets $\gamma_{3}$ in three points, hence its image $C_{4}^{\prime}$ consists of three generators of $R_{5}^{\prime}$ and of a line $l^{\prime}$ in $\pi^{\prime}$.

The $(1,1)$ correspondence between the points of two associated planes $\pi, \pi^{\prime}$ is a collineation.

Now consider the homaloidal system defined by $\pi_{1} \cdots \pi_{5} R_{5}$ in $(x)$, and its inverse $\pi_{1}^{\prime} \cdots \pi_{5}^{\prime} R_{5}^{\prime}$ in $\left(x^{\prime}\right)$ and a second one in $\left(x^{\prime}\right)$ having $R_{5}^{\prime}$ as before, but a system of transversal planes $\bar{\pi}_{i}$, which may be distinct from $\pi_{i}^{\prime}$ or partly identical, and its inverse $\pi_{1}^{\prime \prime} \cdots \pi_{5}^{\prime \prime} R_{5}^{\prime \prime}$ in $\left(x^{\prime \prime}\right)$. By means of the product of the associated Cremona transformations, a plane $\pi$ meeting $R_{5}$ in $\gamma_{3}$ is transformed into a plane $\pi^{\prime \prime}$ meeting $R_{5}^{\prime \prime}$ in a $\gamma_{3}^{\prime \prime}$, and the $(1,1)$ correspondence between these planes is a collineation. Similarly for any number of components. The resulting product will always be of the form

$$
S_{3} \sim \phi_{3 v+1}: R_{5}^{\nu} \pi_{1}^{\rho_{1}} \cdots \pi_{p}^{\rho_{p}},
$$

and the inverse will be of the same form.

Now the question arises whether every Cremona transformation of this form in $S_{4}$ can be expressed as the product of $T_{4,4}$ of the type just considered. An arbitrary $S_{3}$ meets $R_{5}$ in $C_{5}$, $p=1$; the trisecants of $C_{5}$ form a ruled surface $\sigma_{5}$ of order 5 ,

* F. P. White, loc. cit. 
having $C_{5}$ for complete double curve. The image of each of these generating trisecants is a line, and these image lines describe a ruled surface $\sigma_{5}^{\prime}$ having a double curve $C_{5}^{\prime}$ in $S_{3}^{\prime}$ (not the image of $S_{3}$ in $\left.T_{4,4}\right)$. A $(1,1)$ correspondence between the points of $S_{3}$, $S_{3}^{\prime}$ can be established as follows: from any point $P$ in $S_{3}$ can be drawn one and only one trisecant of $R_{5}$. (White. ${ }^{*}$ ) If $P$ is not on $\sigma_{5}$, its associated trisecant $t$ is not in $S_{3}$. The image $t^{\prime}$ of $t$ meets $S^{\prime}$ in $P^{\prime}$. Let $P$ describe a line $p$ in $S_{3}$. It will meet 5 lines of $\sigma_{5}$. Construct the ruled surface of lines $t$ for each position of $P$ on $p$, get its transform in $T$, then cut the transform by $S_{3}^{\prime}$. The section will consist of the image of $p$, and of five generators of $\sigma_{5}^{\prime}$.

Since $p$ is a simple directrix line on the ruled surface of trisecants, its order is one greater than the number of generators in any $S_{3}$ through $p$. Since any $S_{3}$ through $p$ meets $R_{5}$ in $C_{5}$ and contains a surface $\sigma_{5}$ of trisecants, the surface is of order 6 . Its image in $T$ is therefore of order $6(3 \nu+1)$ and in $S_{3}$ the image of $p$ is a curve of order $18 \nu+1$, containing $C_{5}^{\prime}$ to multiplicity $6 \nu$ and lines $\omega_{1}, \cdots, \omega_{p}$ to multiplicities $6 \rho_{i}$ :

$$
p \sim C_{18 \nu+1}: C_{5}^{C^{6} \omega_{1}^{\rho_{1}^{\prime}} \cdots \omega_{p^{\prime}}^{\rho^{\prime}}}
$$

For $\nu=1$ this is a particular case of the transformation belonging to a tetrahedral complex. The $C_{10}$ of $p=11$ of the linear complex is replaced by $C_{5}, p=1$, and 5 of its trisecants. (Sharpe and Snyder. $\dagger$ )

By repeating the argument already used, and using the relations analogous to those used by Montesano, $\ddagger$ the condition $\rho_{1}+\cdots+\rho_{5} \geqq 3(\nu+1)$ can be established; hence by using the $T_{4,4}$ determined by those $\pi_{i}$ of highest multiplicity, the order of the primals of the homaloidal net can be reduced. The transformation $T$ can be expressed in terms of $T_{4,4}, \cdots$.

An $S_{3}$ meets $\phi_{i}, \phi_{k}$ of the system in quartic surfaces through $C_{5}$ and 5 of its trisecants. The residual intersection is $C_{6}, p=3$, meeting $C_{5}$ in 5 points and the 5 trisecants each in three. Hence the surface $f$ thus meets each of the five plane $\pi_{i}$ in cubic curves, and $R_{5}$ in a composite quintic curve consisting of 5 generators.

* F. P. White, loc. cit.

$\dagger$ F. R. Sharpe and V. Snyder, Transactions of this Society, vol. 21 (1920), pp. $52-78$.

$\ddagger$ D. Montesano, Naples Rendiconti, loc. cit. 
Two surfaces $f$ meet in 36 points. The point $\pi_{i j}$ is double for each primal, hence quadruple on their surface of intersection. Of these, three are accounted for by the fact that $\pi_{i j}$ lies on $\pi_{i}, \pi_{i}, R_{5}$; hence $f$ passes through $\pi_{i j}$ simply.

The cubic curve in which $f_{6}$ meets $\pi_{i}$ passes through the four points $\pi_{i j}$ which lie in this plane, so that two such cubics have five intersections at points not coinciding with $\pi_{i j}$. Two $f$ 's, then, have $10 \pi_{i j}, 5$ other points in each of the planes $\pi_{i}$, and one variable intersection.

A plane $\omega$ meets $R_{5}$ in 5 points, and meets each $\psi_{i}$ in a cubic curve $c_{3}$ belonging to the pencil $4 \pi_{i k} 5 \Gamma$. The image $f_{6}^{\prime}$ of $\omega$ contains 5 lines $g^{\prime}$ of $R_{5}^{\prime}$ and meets $\pi_{i}^{\prime}$ (image of $\psi_{i}$ ) in a cubic curve $c_{3}^{\prime}$, in $(1,1)$ correspondence with $c_{3}$. The lines $g^{\prime}$ pierce $\pi_{i}^{\prime}$ in 5 points $\Gamma^{\prime}$, all on $c_{3}^{\prime}$. But these points also lie on $\gamma_{3}^{\prime}$, section of $R_{5}^{\prime}$ by $\pi_{i}^{\prime}$. Through the five lines $g_{1}^{\prime}, \cdots, g_{5}^{\prime}$, in which $f_{6}^{\prime}$ meets $R_{5}^{\prime}$, pass $\infty^{1}$ surfaces $f_{6}^{\prime}$.

The five points $G^{\prime}$ in which any $f_{6}^{\prime}$ meets $\gamma_{3}^{\prime}$ in $\pi_{i}^{\prime}$ apart from the points $\pi_{i k}$ are base points of a pencil of cubic curves. Let $\omega$ meet $R_{5}$ in 5 points $G$. Through each point $G$ passes a generator $g_{i}$ of $R_{5}$. These lines $G g_{i}$ are met by $\infty^{1}$ planes $\omega$. Two such planes have for images surfaces $f_{6}^{\prime}$ having 10 points $\pi_{i k}$ and 5 lines $g^{\prime}$ in common.

6. Case $n=5$. Let $\sigma_{1}, \cdots, \sigma_{6}$ be six three-spaces in [5]. They determine a homaloidal system of order 5 . Any five of the $\sigma_{i}$, as $\sigma_{1}, \cdots, \sigma_{5}$, determine a quartic primal $\psi_{6}$, and similarly for five others. These are images of the base system $\sigma_{i}^{\prime}$ in another [5]. Consider $\sigma_{1}, \cdots, \sigma_{4}$ common to $\psi_{5}, \psi_{6}$. Any two of them intersect in a line, thus defining six lines $l_{i k}$, three skew lines in each of the spaces $\sigma_{1}, \cdots, \sigma_{4}$. The lines are all double lines on the primals of the system; the [3] determined by $\sigma_{12}, \sigma_{34}$ lies entirely on $\psi_{5}, \psi_{6}$ and similarly for the other two [3] determined respectively by $\sigma_{13}, \sigma_{24} ; \sigma_{14}, \sigma_{23}$. The section of $\psi_{5}, \psi_{6}$ consists of the four spaces $\sigma_{1}, \cdots, \sigma_{4}$, the three transversals just described, and a residual ruled variety of order nine, common to all the homaloids of the system.

The base of the $\infty^{5}$ homaloidal system is a three-way variety of order 9 , locus of the $\infty^{2}$ lines that meet all six three-spaces of the bases $\sigma_{i}$.

7. Case $n$ General. This system is always included among those 
determined by a system of bilinear equations; hence all the configurations are expressed by the vanishing of determinants of a matrix. We may write

$$
\sum a_{i k}^{(r)} x_{i} x_{k}^{\prime}=0, \quad(r=1, \cdots, n) .
$$

If $\sum_{i=1}^{n+1} a_{i, k}^{(r)} x_{i}=A_{k}{ }^{(r)}$, then $\sum A_{k}{ }^{(r)} x_{k}^{\prime}=0$, and the birational transformation is expressed by $x_{p}^{\prime}=\left\|A_{k}{ }^{(r)}\right\|,(k \neq p)$, and similarly $x_{p}=\left\|A_{k}^{\prime}(r)\right\|,(k \neq p)$. The primals $\left\|A_{k}{ }^{(r)}\right\|$ are of order $n$, and have in common a manifold $M$ of dimensionality $n-2$ and of order $n(n+1) / 2$, consisting of the $n+1$ spaces $[n-2]$ and of a ruled variety of order $(n+1)(n-2) / 2$.

The formula given by Veneroni* and repeated in the Encyklopädie (vol. III, Chapter 7, p. 967) does not give the base, but the entire intersection. For further general properties, see $M$. Mikam. $\dagger$

Any primal of order $n$ through $M$ belongs to the system. The transversals of the primals in pairs include $(n-2)(n-3) / 2$ spaces analogous to $l_{12}, l_{24}$, etc.

An $[n-r]$ of $[n]$ defined by $r n$-primals of $[n]$ is transformed into a manifold of dimensionality $n-r$ of order ${ }_{n} C_{n-r}$, having with $M$ a manifold $N$ of dimensionality $n-r-1$ and of order $\left(r(n+1) /(n-r){ }_{n} C_{n-r-1}\right.$ including the $n+1$ intersections with the base. The lines which meet the composite $M$ in $n$ points generate a ruled primal of order $n^{2}-1$, the Jacobian of the Cremona transformation. No line meets $M$ in more than $n$ points. (Segre, $\ddagger$ Godeaux. $\S$ )

8. Related Questions. A number of questions closely related to those here considered have been solved recently. Thus, the algebraic surfaces on a cubic primal of $S_{4}$ (Archbald $\|$ ); octavic normal surfaces with a double line in [5] (Babbage 1 ); isolated singular points in the theory of algebraic surfaces (Babbage ${ }^{* *}$ ); some chains of theorems derived by successive projection

* Loc. cit.

$\dagger$ M. Mikam, Prag Ceske Akademie Rozpravy, vol. 39 (1929), No. 21.

$\ddagger$ C. Segre, loc. cit.

$\$$ L. Godeaux, loc. cit.

II J. W. Archbald, Proceedings of the Cambridge Philosophical Society, vol. 27 (1931), pp. 405-406, and vol. 29 (1933), pp. 484-486.

I D. W. Babbage, ibid., vol. 29 (1933), pp. 95-102 and 405-406.

** D. W. Babbage, ibid., vol. 29 (1933), pp. 212-230. 
(Lob*). Segre's theorem concerning curves on ruled surfaces has been considered and extended by Welchman. $\dagger$ A useful scheme for mapping spaces $S_{k}$ of an $S_{m}$ upon the points of a third of order $(m-k)(k+1)$ is given by Room, $\ddagger$ and Babbage gives a helpful discussion of the transformations of certain surfaces having special singularities. Welchman $\S$ has generalized certain incidence formulas to apply to incidence scrolls, ruled surfaces generated by certain directrix spaces. It would be well to extend this investigation to apply to varieties generated by planes, [3], by similar methods. (Welchman.\|)

A discussion of the forms and possible transformations of isolated singular points of algebraic surfaces, extending the work of Godeaux, ๆ was made by Babbage. ${ }^{* *}$ Roth $\dagger \dagger$ gives further examples of surfaces in hyperspace containing triple curves and obtains some further results concerning composite surfaces contained within a given system in higher spaces. $\sharp \ddagger$

Six lines of $S_{3}$ can have various relations to each other discussed by Todd, $\$ \S$ generalizing Ursell's |||| study of seven lines on a quartic surface, the results of Richmond, $\Phi$ and the earlier paper by Wakeford.***

A detailed analytic account of the $\infty^{n-2}$ lines generating the $V_{n-1}$ in $[n]$ is given by Eiesland. $\dagger \nmid \dagger$ These manifolds are the

* H. Lob, Proceedings of the Cambridge Philosophical Society, vol. 29 (1933), pp. 45-51.

† W. G. Welchman, ibid., vol. 29 (1933), pp. 382-388.

$\ddagger$ T. G. Room, ibid., vol. 29 (1933), pp. 331-336.

\& W. G. Welchman, ibid., vol. 29 (1933), pp. 235-254.

\| W. G. Welchman, Proceedings of the Cambridge Philosophical Society, vol. 29 (1933), pp. 103-115.

I L. Godeaux, Bulletin de l'Académie Royale de Belgique, Classe des Sciences, (5), vol. 15 (1929), pp. 317-318.

** Loc. cit.

†† L. Roth, Proceedings of the London Mathematical Society, (2), vol. 30 (1929), pp. 118-126; Proceedings of the Cambridge Philosophical Society, vol. 28 (1932), pp. 300-310, and vol. 29 (1933), pp. 178-183.

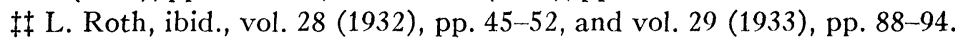

$\S \S$ J. A. Todd, ibid., vol. 29 (1933), pp. 52-68.

||| H. D. Ursell, ibid., vol. 25 (1929), pp. 31-38.

१ १ H. W. Richmond, Journal of the London Mathematical Society, vol. 7 (1932), pp. 113-117.

*** E. K. Wakeford, Proceedings of the London Mathematical Society, (2), vol. 21 (1933), pp. 89-113.

†† J. Eiesland, Palermo Rendiconti, vol. 54 (1930), pp. 335-365. 
single $[n-2]$ of the base primes. He gives much more information concerning questions discussed by Wong.* Particular attention is given to $n=5$. Various particular cases are included. Another derivation of the equation of $V_{n-1}^{n-1}$ is given by Rupp. $\dagger$

9. Transformations Defined by Projective Systems. An important type of Cremona transformations, applicable to spaces of any number of dimensions and including a large number of cases in each, can be illustrated by the simplest case as follows.

Given four points $A, B, C, D$ in the plane, and a line $d$ in the plane, not passing through any of them. Make the conics of the pencil projective with the points of $d$. A point $y$ fixes a conic and a point $Z$ on $d$. The line $y Z$ meets the conic belonging to $Z$ in a point $y^{\prime}$. The correspondence $y, y^{\prime}$ is an involutorial Cremona transformation of the type $c_{1} \sim c_{7}: 4^{3} 3^{2}$ in which the three double points are all on $d$. They are defined as those points $Z$ which lie on the conics associated with them. When one of these conics is composite, the component through $Z$ divides out, lowering the order of the transformation. In the plane, all of these are particular cases of the Geiser involution. Interesting variations occur when the four points approach each other in various ways. The projectivity may be replaced by a $(1, k)$ correspondence, $k$ conics being associated with the same point $Z$.

In space, the simplest case is that of a pencil of quadrics, the base curve not meeting the line $d$. Among the possible types are those discussed by Montesano, $\ddagger$ furnishing an illustration of a congruence of lines being transformed into itself. The pencil of quadrics may be any of the possible types, and the correspondence may be $(1,1)$ or $(1, k)$. The interesting features are the appearance and behavior of parasitic lines.

The quadrics may be replaced by a pencil of surfaces of order $n$ having $d$ to multiplicity $n-2$. When the residual curve is nonreducible, these cases have all been considered by Carroll. $\S$ The application to pencils having a composite residual base curve has thus far been limited to pencils of cubics having $d$ as a simple base line.

* B. C. Wong, this Bulletin, vol. 34 (1928), pp. 829-832.

$\dagger$ C. A. Rupp, this Bulletin, vol. 35 (1929), pp. 319-320.

$\ddagger$ D. Montesano, Giornale di Matematiche, vol. 31 (1893), pp. 36-50. 717.

$\S$ E. T. Carroll, American Journal of Mathematics, vol. 54 (1932), pp. 707- 
The configuration of the homaloids along the various base lines includes an unexpectedly large number of variations. (Rusk.*) It would be well worth while to make the corresponding study of pencils of surfaces of larger order, and to extend it to pencils of primals in hyperspace.

The straight line $d$ may be replaced by a rational curve. Here the possible number of types is limited, but new arrangements of fundamental elements appear. The case of an irreducible residual base curve has been completed, and that of composite curves is limited to the features characteristic of the composite case. (Black. $\dagger$ ) Various other types have been considered by Davis, $\ddagger$ in connection with Cremona transformations contained in a special or non-special linear line complex.

Isolated cases had been discussed earlier by Pieri and by Montesano. The latter proposed to classify involutions according to the product of the order of the complex to which each belongs and the number of pairs of conjugate points on each line. The involution belonging to the general cubic primal of $S_{4}$ belongs to a special linear complex, and each line has two pairs; it is probably irrational, but no case has as yet been proved definitely irrational. Illustrations defined by pairs of planes in a curiously simple form were given by Snyder. $\$$ All of these cases are rational. (Dye.\|) Since every line of a surface of a pencil that passes through its associated point is a fundamental line of the second kind, a particularly instructive case is that in which every surface of the pencil is ruled. Through every point of the rational curve $d$ pass a number of generators, hence the surface generated by them is a component of every homaloid. The base curves of the pencil and isolated generators form the entire system of fundamental curves. Along the directrix curves the surfaces have contact of maximum order, furnishing a prob-

* Evelyn T. Carroll $\equiv($ Mrs.) E. C. Rusk, American Journal of Mathematics, vol. 56 (1934), pp. 96-108.

$\dagger$ A. H. Black, Transactions of this Society, vol. 34 (1932), pp. 795-810; this Bulletin, vol. 40 (1934), pp. 417-420.

$\ddagger$ H. A. Davis, American Journal of Mathematics, vol. 52 (1930), pp. 58 71 , and vol. 53 (1931), p. 72; H. A. Davis and Tyr. Davis, Tôhoku Mathematical Journal, vol. 33 (1931), pp. 53-58.

$\S$ V. Snyder, this Bulletin, vol. 36 (1930), pp. 89-93; Atti, Bologna Congress, vol. 4 (1930) [1928], pp. 13-21.

|l L. A. Dye, Transactions of this Society, vol. 32 (1930), pp. 251-261. 
lem in postulation and equivalence not accounted for by known formulas. Apart from a few isolated cases, all of these types have for directrix system a line and a rational curve of order $m$ meeting it in $m-1$ points. (Snyder.*)

A non-linear null system defined by a cubic surface gives rise to a Cremona involution by associating each surface of a pencil of cubics with the points of a line, each surface passing through its associated point but not containing the line. (Snyder and Schoonmaker. $\dagger$ ) Applications of the method to obtain certain space generalizations of the Geiser and Bertini plane involutions have been made by Dye and Sharpe. $\ddagger$

10. Double Fives. B. Segre $\$$ considers the problem: Is it possible to find a pyramid (simplex) which is simultaneously inscribed in and circumscribed to a quadric primal $Q$ in $S_{4}$ ? Let the faces $\alpha_{i}$ touch $Q$ in $B_{i}$, and four faces meet in $A_{i}$. If such a simplex is possible, then both pyramids $A_{1} \cdots A_{5}$ and $B_{1} \cdots B_{5}$ are both inscribed and circumscribed, and are polar conjugates as to $Q$. It is shown that pyramids do exist. A face (three space) $\alpha_{5}$ cuts $Q$ in a point cone, vertex at $B_{5}$, and containing the lines $B_{5} A_{i},(i=1,2,3,4)$. On the cone these four generators are equianharmonic. Two pairs of conjugate pyramids are projectively equivalent. One pair is transformed into the other by 120 collineations and by 120 dualities. Together these form a group under which $Q$ remains invariant.

Five associated planes of $S_{4}$ (as defined by C. Segre\|) always admit a pair of conjugate pyramids, and consequently define the quadric $Q$. The five planes are the intersections of the five pairs of opposite faces. By polarity as to $Q$ these are changed into five associated lines, related to the given planes. These lines are the joins of associated vertices of the two conjugate pyramids. If $Q$ be projected stereographically from a point $L$ upon it into an $S_{3}$ not passing through $L$, a fundamental conic $\gamma$ results. The twenty lines that are projections of $A_{i} B_{k},(i \neq k)$, are all unisecants of $\gamma$.

* V. Snyder, Transactions of this Society, vol. 35 (1933), pp. 341-347.

$\dagger$ V. Snyder and H. E. Schoonmaker, American Journal of Mathematics, vol. 54 (1932), pp. 299-304.

$\ddagger$ L. A. Dye and F. R. Sharpe, Transactions of this Society, vol. 36 (1934), pp. 292-305.

§ B. Segre, Lincei Memorie, (6), vol. 2 (1928), pp. 204-209.

\| C. Segre, Torino Memorie, (2), vol. 39 (1903), pp. 1-48. 
The projections of the points $A_{i}, B_{i}$ form a double five of points related to the conic. If $\gamma$ is projected from any one of them, the resulting quadric cone contains four other points of the double five, and on the cone the generators containing them are always equianharmonic. An irreducible conic has $\infty^{10}$ related double fives of points.

11. Sets of Five Lines in $S_{3}$. If now the well known mapping of the lines of $S_{3}$ on the points of an irreducible quadric primal of $S_{5}$ be employed, corresponding to double fives of points relative to the various hyperplane sections of $Q$, there exist configurations of lines in $S_{3}$ which have the following properties.

Let $a_{i}, b_{i}$ be two sets of five lines each in $S_{3}$ such that (1) they all belong to the same non-special linear complex $L,(2)$ that each line $a_{i}$ is incident to four lines $b_{k}$, but not to $b_{i}$, and each line $b_{i}$ is incident with all lines $a_{k}$ except $a_{i}$. Such a configuration of lines is called a double five. The four points in which $a_{i}$ meet $b_{k}$ are equianharmonic.

Given a line $b_{5}$ in a non-special linear complex $L$, and four lines $a_{1}, \cdots, a_{4}$ of $L$ meeting it, the reguli defined by these lines $a_{i}$, three at a time, contain one and only one line of $L$, apart from $b_{5}$. The necessary and sufficient condition that the four lines thus obtained have only one common transversal is that the set of lines $a_{i}$ be equianharmonic.

A number of interesting properties follow from this simple construction of a double five of lines in $S_{3}$. This result was obtained in terms of lines of $S_{3}$ by B. Segre in an earlier paper.* The same result was obtained by Weitzenböck. $\dagger$ (See also Schaake. $\ddagger$ ) The general properties of double fours and of double fives were given by Maurer. $\$$

12. Sets of Oriented Circles in the Plane. Another interpretation can be given in terms of oriented circles in the plane, mapping each on a point of a general quadric $Q$ primal of $S_{4}$, in such manner that those oriented circles which touch each other in the same point are mapped on the points of a line of $Q$. In a plane are $\infty^{10}$ double fives of oriented circles, such that each circle is

* B. Segre, Rendiconti dei Lincei, (6), vol. 2 (1925), pp. 539-542.

$\dagger$ R. Weitzenböck, Amsterdam Proceedings, vol. 31 (1928), pp. 133-137.

$\ddagger$ G. Schaake, Amsterdam Proceedings, vol. 31 (1928), pp. 715-717.

$\S$ A. Maurer, Bonn disseration, 1929. 
touched by 4 circles, belonging to the quintuple to which the given circle does not belong. Upon the latter circle the four points of contact are equianharmonic. This last property was found by a different method by Barbilian,* and further discussed by Tzitzeica. $\dagger$ The relations between the two interpretations were shown by Weiss. $\ddagger$ Part of these relations were obtained by Study, $\S$ who also obtains a double five, but these are not equivalent to those here considered, as the complex containing the ten lines is so particularized that the $S_{4}$ represented by it in $S_{5}$ cuts $Q$ in a quadric $Q^{\prime}$ having the property that all ten lines touch it.

Gambier\| applies the ideas of orientation to circles and spheres in contact, including a metric generalization of the Morley-Peterson property to $S_{n}$; he $\int$ makes an application to orthogonal systems by methods of pure geometry, the case $n=4$ producing the results obtained by Barbilian. E. Ciani** points out that the whole idea of double fives is due to him. $\dagger \dagger$

Cornell University

* D. Barbilian, Bucharest Akademie Bulletin, Mathematics and Physics, vol. 1 (1929), pp. 12-16.

$\dagger$ G. Tzitzeica, Bucharest Akademie Bulletin, Mathematics and Physics, vol. 1 (1929), pp. 16-21.

$\ddagger$ E. A. Weiss, Amsterdam Proceedings, vol. 35 (1932), pp. 969-978.

$\S$ E. Study, Berlin Sitzungsberichte, 1926, pp. 360-380.

|| B. Gambier, Comptes Rendus, vol. 190 (1930), pp. 157-159.

đB. Gambier, Bulletin des Sciences Mathématiques, vol. 55 (1931), pp. 75-96; Journal de Mathématiques, (9), vol. 11 (1931), pp. 377-387.

** E. Ciani, Annali di Matematica, (3), vol. 8 (1902), pp. 1-21.

t† E. Ciani, Rendiconti dei Lincei, (6), vol. 17 (1933), pp. 215-217. 